\title{
REVIEW ARTICLE \\ Reasons for optimism in the search for new vaccines for tuberculosis
}

\author{
T. G. EVANS*† \\ Vaccitech, Oxford, UK \\ Received 8 December 2016; Final revision 25 February 2017; Accepted 7 March 2017; \\ first published online 17 April 2017
}

\section{SUMMARY}

In the development of vaccines for tuberculosis (TB), the combination of the will, funding, scientific rigor, new tools, refined animal models and improved clinical trial designs are all converging at an opportune moment. The lack of optimism that has surrounded the likelihood for finding novel TB vaccines has resulted from a lack of correlates of vaccine-induced protection, a lack of tool candidate vaccines to probe the immunologic space, which may be needed, and the negative result of one recent trial. A vaccine for TB that can be delivered at a reasonable cost to the marketplace will have greater impact on the incidence of new cases of TB than any intervention in world history. Now is the time to increase resources, both financial and human intellectual capacity, for a global tuberculosis vaccine effort.

Key words: BCG vaccination, tuberculosis (TB), vaccine safety.

Tuberculosis (TB) is now the leading single pathogen cause of death in the world. In 2015, there were an estimated 1.8 million deaths from TB, making it the number nine overall cause of mortality worldwide. Nearly one billion people have died of TB over the past few centuries, an astounding number, and TB is a leading cause of mortality in human immunodeficiency virus (HIV)-infected individuals and in women of childbearing age. Ninety-nine percent of the TB deaths and $95 \%$ of the over 10.4 million new cases each year occur in the low- and middle-income countries that comprise $85 \%$ of the world's population. One-third of the world's population is estimated to be latently infected and $5-10 \%$ of these people may

\footnotetext{
* Author for correspondence: T. G. Evans MD, Vaccitech, Oxford, UK.

(Email: tomevansmd@gmail.com)

$\dagger$ This Review is a written summary of the Heath Clark Lecture given in June 2016 at the London School of Hygiene and Tropical Medicine.
}

go on to develop active TB disease (WHO: Global Tuberculosis Report 2016).

In 2014, the WHO prioritized R\&D as one of the pillars for their plan to reach the goals set for 2035 to reduce TB deaths by $95 \%$ and to reduce new infections by $90 \%$. The reductions targeted assume an unlikely $10 \%$ decline per year over the next decade in the incidence of new active TB cases (up from the present value of approximately $2 \%$ decline per year), and the implementation of new tools by the year 2025. The only tools that likely will have an impact on the epidemic will be: (1) the use of new diagnostics that allow for earlier case-finding; (2) a tool to identify the subset of the latent population at highest risk for active TB development to receive preemptive therapy; or (3) a new vaccine that can be used in a mass vaccination campaign. Although new drugs are needed to overcome the increasing issue of drug resistance, the new therapies for TB (even those that shorten treatment) are highly unlikely to contribute in any appreciable manner to reach the WHO 2035 goals [1]. 
Despite the greater number of new infections and death from TB than HIV, approximately seven times as many funds are allocated to HIV vaccine research than to TB vaccines, and the amount spent on new drugs has averaged 1.5-2 times that spent on vaccines over the last decade (Treatment Action Group 2015 Report). One of the reasons for the low funding of TB vaccine research has been a pessimistic view of the likelihood of finding a new TB vaccine; the more optimistic counterargument follows.

The entrenched pessimism can be summarized in a few central issues. A first major criticism is that we have no correlate of protection from TB after nearly a century of use of Mycobacterium bovis bacillus Calmette-Guérin (BCG), the only vaccine available to (partially) protect against TB disease [2]. BCG is a live, attenuated vaccine that is widely administered to infants in most areas endemic for TB. BCG has been shown to be effective for the prevention of more serious extrapulmonary TB in young children, such as tuberculous meningitis and miliary TB. A meta-analysis of prospective trials and case-control studies concluded efficacy against pulmonary TB to be approximately $50 \%$, with a range from a low of zero to a high of $80 \%$ [3]. Multiple attempts to delineate a correlate of protection using BCG have not been fruitful, but have led to a better understanding a correlate of risk [4, 5]. In short, BCG immune responses to date have not been able to guide vaccine development, as other first generation vaccines have done later vaccine development for other diseases.

Nonetheless, until the last decade, no novel vaccine had ever been developed with an a priori knowledge of the correlate of protection, and indeed a correlate can only be validated once a vaccine is shown to be protective. The assumption that the correlate of protection using BCG in infants, mainly to prevent disseminated disease, will be the same for that in adolescents or young adults, may not be justified. For example, the correlate of protection for Flumist in infants appears to be different than that using inactivated flu vaccine in adults [6]. There is no question that finding a correlate of vaccine-induced protection will speed present and future vaccine development, derisk the field for companies to invest, and allow faster adaptation of vaccines to different geographic areas, but is not a given necessity in vaccine product development.

A central concept that has emerged in the last decade is that TB pathogenesis in the non-human primate (NHP) model is sufficiently similar to that in humans that once protection is observed in the NHP, then correlates of protection from those candidates can guide decisions on advancing candidates through various stages of clinical trials [7]. While this is a reasonable and attractive strategy, the approach has several shortcomings. First, to prove that the correlate is truly associated, confirmatory NHP testing of human results (both positive and negative) will be needed, which in general will require 9-12 months of further study and 1.5-2 million dollars of expense. Second, the animal TB infection model at present utilizes bronchial instillation with a non-encapsulated organism, which does not reflect the natural human form of transmission [8]. Third, the NHP model is still not validated, even for BCG, and the present model using low dose inoculation in cynomolgus macaques has not yet been used to show a negative result with the vaccine candidate known as MVA85A, the only candidate with clear efficacy data in the last decade. Validation requires showing both negative and positive predictive correlations, and thus testing MVA85A to show a negative result in cynomolgus macaques should be a priority, if this NHP low-dose model will be used as the major stage gate to further Phase $2 b$ trials.

The lack of a small animal model that can clearly recapitulate TB has been seen as a major hindrance to developing TB vaccines, and yet many diseases lack any small animal model upon which to base product development. TB is actually endowed with better mouse and rodent models, especially the guinea pig, than many competing fields, especially for viruses that are non-pathogenic in mice. Thus, although the mouse may not recapitulate the human diseases, it can be used to determine whether a biologic effect of certain vaccine strategies may impact the growth of the Mycobacterium tuberculosis (MTb) bacterium [9]. If the immune mechanism is similar in a mouse and human, then the use of the mouse model to show biological activity is a reasonable first step.

Another factor in the pessimism for vaccine development lies in the fact that because MTb has co-evolved with humans over many years and may subvert the human immune system to maintain its propagation [10], some scientific skepticism has arisen concerning the prospect for developing an effective vaccine. Additionally, prior active pulmonary $\mathrm{TB}$, in contrast to protective latent $\mathrm{TB}$, may actually be a risk factor for disease after reinfection. However, many other diseases caused by pathogens that harbor large and complex genomes that are designed to elude or hijack the human immune system and have a long 
co-evolutionary history with man have been controlled by vaccination; e.g. smallpox, varicella, pertussis, and cholera. Given that a 'natural' immune response may not protect against $\mathrm{TB}$, it may be necessary to pursue approaches that induce 'non-natural' immunity. Examples of such approaches include the use of tetanus toxoid, the success of polysaccharide conjugate vaccines, and the new findings of the role of non-MHC (major histocompatibility complex) class I -restricted protection in the SIV (Simian immunodeficiency virus) NHP model [11, 12].

Another source of negativity was the 'failure' of the first efficacy trial of a novel TB vaccine, a Phase $2 b$ study that evaluated the use of the modified vaccinia Ankara (MVA) Ag85A vaccine to boost immunity in infants when given 4 months after neonatal BCG [13]. However, the trial had a negative outcome, but was not a 'failure', as the hypothesis stated was answered by the trial design and conduct. A 'failed' trial is one in which the outcome is uncertain due to the poor conduct of the study, underpowering due to improper assumptions, or inconclusive results. This single definitive negative result should not hamper further vaccine development, and many positive outcomes resulted from the study: the consensus on endpoints for such a trial [14], the building of site capabilities to conduct a large trial, the demonstration that a successful Phase $2 \mathrm{~b}$ TB registrational study could be carried out in infants [15], and the further understanding of correlates of risk for TB in infants [16]. Whether this disappointing outcome was due to the magnitude of the immunologic response, the single dose of the single antigen $(\mathrm{Ag} 85 \mathrm{~A})$ studied (and chosen from 4000 potential candidates), the population vaccinated, or an incorrect immunologic hypothesis is not clear. Many programs of vaccine development had negative studies during the path to a successful product, and TB will be no exception. Certainly the failure of four large efficacy trials in HIV has not hampered the spending on HIV vaccines. The single marginally positive trial that was derided by experts in the field at time of initiation and gave one glimmer of hope (RV144) has resulted in a recently launched, massive follow-on effort in the 702 trial in South Africa [17, 18].

There are multiple reasons to believe that a novel and efficacious vaccine for TB is likely to be developed during the next few decades of effort. First, the human immune system can contain TB infection in the majority of cases following infection; i.e. up to $90 \%$ of those infected never develop TB in their lifetime. Second, we already have a partially effective vaccine for infants.
Third, there is substantial evidence that prior latent MTb infection may protect against later disease [19]. Fourth, there is a solid understanding of which immunologic pathways control TB through the risk associations that can be inferred from Medelian susceptibility $[20,21]$. Fifth, the tools to measure immunologic correlates of protection in animal models have markedly improved, as has our ability to translate those findings from the NHP to man.

The development of an effective vaccine requires a clear target, and that has also been difficult for TB, with an initial focus on the use of infant vaccination based on harmonization with the WHO EPI strategy. However, infants make up only a minor fraction of the disease burden and are not responsible for almost any of the ongoing transmission. Expert modeling by independent groups in TB prevention and control agrees that the largest global vaccine impact would result from mass vaccination of all adolescents/young adults in high-burden countries, regardless of their infection status, even with a vaccine that is only $50-60 \%$ efficacious over a 10-year duration [22-24]. Such a vaccine strategy could prevent up to 50 million cases of incident TB cases in high-burden settings during the first 35 years after its introduction, and would be effective in preventing infant disease as well. This impact would likely save millions of lives and billions of dollars in treatment and control costs. It is also important to note that strains of $\mathrm{MTb}$ that are resistant to standard anti-tuberculous drugs, including MDR (multiply drug-resistant) and XDR (extensively drug-resistant) strains are becoming more common. While MTb is likely to continue to evolve resistance against drugs develop to combat it, it is highly unlikely that such resistance would result in a concomitant development of resistance against otherwise effective TB vaccines.

There are other reasons for optimism. The tools that are needed to evaluate different vaccine candidates and approaches are now being funded and developed. This includes methods to determine bacterial burden in animals (and potentially in man) using bacteria expressing sensitive soluble or volatile makers that will allow quantification of the number of bacteria down to a total of $10^{3} \mathrm{CFU}$ (Fortune, personal communication). Such quantitation will allow developers to measure the impact of vaccines on clearing bacteria either after infection, or during latency, as compared with placebo, and determine whether there is a measurable biologic effect (in essence, a proof of concept). Use of bar-coded strains can also be employed to show a decrease in the 'founder' 
population size after infection, and thus account for early events that result in clearance of a proportion of the infecting inoculum [9].

The use of new Positron emission tomography (PET) imaging techniques may allow assessment of both the degree of inflammation, as well as the potential quantification of pathogen load [8, 25-28]. Such methods could potential shorten the time for NHP studies from over 16-20 weeks down to 6-8 weeks, resulting in both increased speed and cost savings, and a better ability to correlate NHP and human data. The strains that are engineered to allow such sensitive markers can also be modified to allow for conditional replication (using techniques such as tetracycline inducible on-off switches or non-natural amino acids), which will be one of the first steps toward the development of a potential human challenge model. Such a challenge model would allow the rapid testing of a biologic effect of vaccination on bacterial burden, and would allow for Proof of Concept $(\mathrm{PoC})$ studies to be conducted for less than one-tenth the cost of that presently required.

Natural transmission animal models that will allow for evaluation of vaccines that may block the earliest stages of infection are now being developed. Recent work showing that guinea pigs can be infected, if placed downstream from productively infected NHPs, similar to the pioneering studies of Riley using guinea pigs housed downstream from diseased humans (A. Rawkins, personal communication). Such systems can then be used to determine if antibody-based or other vaccines can block the natural, capsule-directed TB bacillus from setting up a productive infection that ultimately leads to disease [29, 30].

The use of low-dose challenge, sophisticated PET/ $\mathrm{CT}$ imaging techniques, and novel vaccine candidates have recently shown that the macaque model may potentially be useful to pose hypotheses of correlates of vaccine-induced protection to be studied in humans. For the first time, we have clear evidence of protection in the very virulent rhesus model using a CMV-based vaccine construct encoding six TB antigens, (L. Picker, personal communication) and further studies using this 'tunable' vaccine strategy are ongoing to delineate the rhesus correlate of protection, including assessment of tissue resident $\mathrm{T}$ cells in the lung $[11,12]$. In the cynomolgus model, protection has been achieved with an adjuvanted fusion protein that is now starting a Phase $2 b$ trial in Tanzania and South Africa [31]. These studies will further our understanding of the value of the macaque model.
Newer systems biology tools have allowed a better understanding of TB risk, and are also being applied to correlates of vaccine-induced protection in the NHP. Gene expression patterns of inflammatory biosignatures have correlated with risk for TB disease progression, and with the extent of radiographic involvement in both active and latent TB cases $[5,32]$. In response to these data, TB vaccine developers are pursuing a systems immunology approach in which gene expression signatures are compared in samples from various time points [33]. These signatures are then correlated to either specific measures of immunogenicity, or to protection in efficacy studies. This method allows for a broader unbiased net to be cast in assessing immune responses, and in searching for a correlate of vaccine-induce protection.

There exists an arsenal of vaccine platform approaches that are ready to be use in human studies to test immunologic hypotheses, if needed [34] (the present vaccine candidates are shown in Fig. 1). These include inactivated whole cell or whole-cell extracts [M. vaccae, DAR-901 (M. obuense)], viralvectored candidates (influenza, MVA, and chimpanzee adenovirus), fusion protein subunits with $\mathrm{TH} 1$-inducing adjuvants (M72/AS01, H4/IC31, H56/IC31, and ID93/ GLA-SE), and live recombinant BCG or attenuated TB vaccines (VPM 1002, Aeras 422, rBCG30, and MTBVAC). To date, clinical trials characterizing these candidate vaccines have included studies of safety and immunogenicity in diverse populations, including healthy, TB-naïve adults and infants, latently infected adolescents and adults, HIV-infected adults, as well as patients undergoing drug treatment for TB. Importantly, in all of these trials there has been no evidence of a 'Koch-like' reaction, even when four different candidates (MVA, human adenovirus 35, M72, and ID93) have been administered to recently treated active TB patients.

In the near future, a number of trials will build the needed knowledge base to inform on the potentially successful or unsuccessful strategies to pursue. A Phase IIB trial in 3600 HIV-uninfected, adults latently infected with MTb is under way in three African countries (South Africa, Kenya, and Zambia) using the GSK M72 adjuvanted fusion protein vaccine. This study should further our understanding of potential correlates of protection, as well as the role of TH-1induced immunological responses in preventing the development of disease in latently infected individuals. Data are expected in 2018. A large 10000 person study to prevent TB disease is underway in Guanxi 


\section{Global Clinical Pipeline of TB Vaccine Candidates}

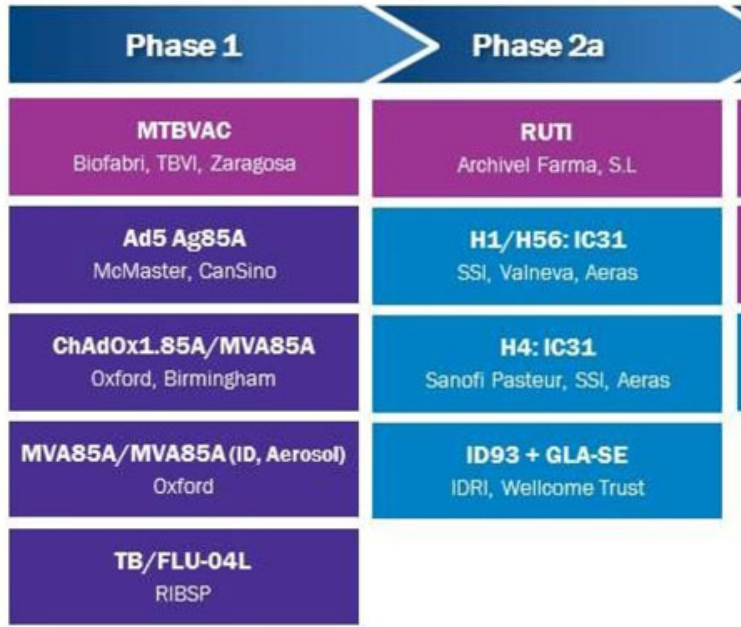

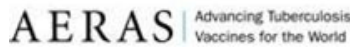

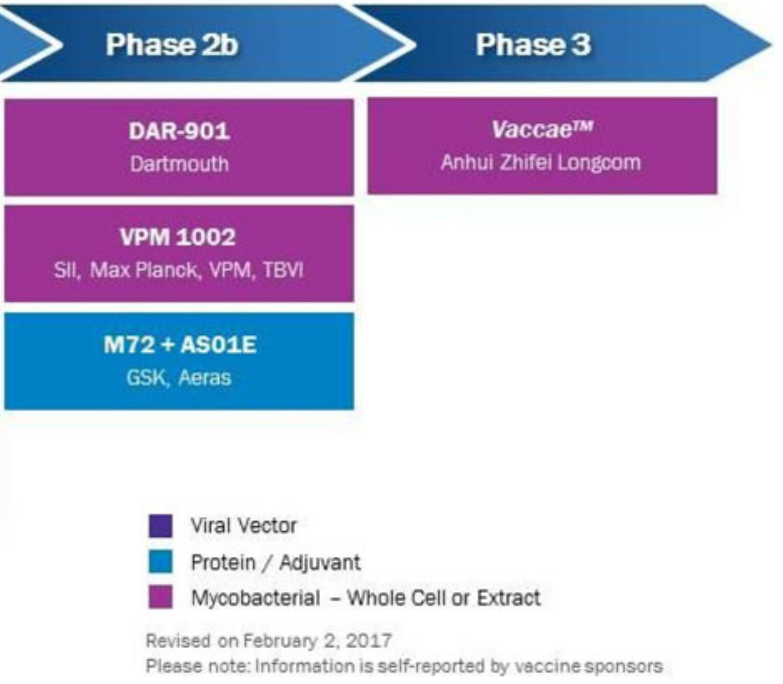

Fig. 1. Global Vaccine Portfolio October 2016.

Province, China using a killed non-tuberculous mycobacterial vaccine given as a series of multiple vaccinations to latently infected adults with PPD reactions greater than $15 \mathrm{~mm}$.

In addition to these large-scale $\mathrm{PoC}$ trials using disease endpoints, a new set of human studies are under way, based on the use of innovative trials designs using more focused populations specifically selected to reduce sample size, and intended to show the biologic activity of vaccine candidates. The first of these new trial designs is testing whether a novel vaccine (H4/IC31) or the use of BCG re-vaccination can prevent sustained infection by MTb (as opposed to disease) [35]. The trial uses novel blood tests in which BCG vaccination does not interfere with the test result - a common obstacle with the longtime, standard diagnostic, the tuberculin skin test. The study is enrolling adolescents in South Africa with a high rate of incident MTb infection, thereby requiring only 330 subjects per arm rather than the 2000 or more needed in the classic PoC trials. Results are expected mid-2017. The second innovative trial design is to study the ability of a vaccine to prevent the $4-6 \%$ relapse and/or reinfection rate typically observed following successful treatment of active TB. A 'Prevention of Recurrence' (POR) trial using the ID93 candidate will likely begin shortly and require approximately 450 subjects per arm, and a similar POR study is being undertaken in India using the recombinant BCG candidate VPM1002. All of these trials represent an attempt to make decisions earlier in development (a 'shift to the left'), given the 30-50 million dollar cost needed to conduct classical efficacy disease endpoint studies [24].

Other innovative leads that will be aggressively pursued over the next 5 years will include the use of aerosolized candidates, either alone or in combination. The protection apparently afforded by latent TB infection of the lung has led to renewed interest in aerosolized whole-cell TB vaccines, and two recent small studies in humans have shown that aerosolization of high dose BCG $10^{5} \mathrm{CFU}$ ) is well tolerated (H. McShane, personal communication). The combination of aerosolized or intramuscular adenoviral vectored vaccines followed by MVA has been especially promising in both preclinical models and in early human trials $[36,37]$. CMV-vectored candidates will likely move forward in both the TB and HIV areas, as they induce prolonged and high levels of effector $\mathrm{T}$ cells in the mucosal location where the pathogen first encounters the human host, although a risk for overactivation is a potential detriment. The value of a number of novel BCG replacement strategies will also become clearer.

To further the likelihood of success, much greater funding will be needed for TB vaccine $R \& D$ than is presently available. The business case for TB vaccines, as recently developed by a working group of Aeras, the Tuberculosis Vaccine Initiative, the Bill and Melinda 
Gates Foundation, the European Commission, the European Investment Bank, is compelling, with potential returns on investment that range from hundreds of millions of dollars to over a billion dollars of annual revenue (available from Areas.org). Due to the perceived scientific risks, however, it has been difficult to engage industry, whether large or small, from entering into this field without substantial early subsidization from granting and governmental organizations.

Why will novel TB vaccines be successful? A combination of the will, funding, scientific rigor, new tools, refined animal models and improved clinical trial designs are all converging at the right time in history. A vaccine for TB that can be delivered at a reasonable cost to the marketplace will have the greatest impact on the incidence of new cases of TB than any intervention in world history. Now is the time to increase resources, both financial and human intellectual capacity, for this effort.

\section{ACKNOWLEDGEMENTS}

Many thanks to the Graham Clark fellowship for allowing the interaction of the author with the members of the TB Centre of the London School of Hygiene and Tropical Medicine, and to the Bill and Melinda Gates Foundation for recognizing the need and supporting the funding of new TB vaccines.

\section{REFERENCES}

1. Fofana MO, et al. Population-level impact of shortercourse regimens for tuberculosis: a model-based analysis. PLOS ONE 2014; 9: e96389.

2. Rodrigues LC, Diwan VK, Wheeler JG. Protective effect of BCG against tuberculous meningitis and miliary tuberculosis: a meta-analysis. International Journal of Epidemiology 1993; 22: 1154-1158.

3. Colditz GA, et al. Efficacy of BCG vaccine in the prevention of tuberculosis. Meta-analysis of the published literature. Journal of American Medical Association 1994; 271: 698-702.

4. Kagina BM, et al. Specific T cell frequency and cytokine expression profile do not correlate with protection against tuberculosis after bacillus Calmette-Guerin vaccination of newborns. Am J Respiratory and Critical Care Medicine 2010; 182: 1073-1079.

5. Zak DE, et al. A blood RNA signature for tuberculosis disease risk: a prospective cohort study. Lancet 2016; 387: 2312-2322.

6. Belshe RB, et al. Correlates of immune protection induced by live, attenuated, cold-adapted, trivalent, intranasal influenza virus vaccine. Journal of Infectious Diseases 2000; 181: 1133-1137.
7. Pena JC, Ho WZ. Monkey models of tuberculosis: lessons learned. Infection and Immunity 2015; 83: 852-862.

8. Scanga CA, Flynn JL. Modeling tuberculosis in nonhuman primates. Cold Spring Harbor Perspectives in Medicine 2014; 4: a018564.

9. Smith CM, et al. Tuberculosis susceptibility and vaccine protection are independently controlled by host genotype. MBio 2016; 7: e01516-16.

10. Comas I, et al. Human T cell epitopes of Mycobacterium tuberculosis are evolutionarily hyperconserved. Nature Genetics 2010; 42: 498-503.

11. Hansen SG, et al. Cytomegalovirus vectors violate CD8 + T cell epitope recognition paradigms. Science 2013; 340: 1237874.

12. Hansen SG, et al. Broadly targeted CD8(+) T cell responses restricted by major histocompatibility complex E. Science 2016; 351: 714-720.

13. Tameris MD, et al. Safety and efficacy of MVA85A, a new tuberculosis vaccine, in infants previously vaccinated with BCG: a randomised, placebo-controlled phase 2b trial. Lancet 2013; 381: 1021-1028.

14. Hatherill M, Verver S, Mahomed H, Taskforce on Clinical Research Issues STBPWGoTBV. Consensus statement on diagnostic end points for infant tuberculosis vaccine trials. Clinical Infectious Diseases 2012; 54: 493-501.

15. Tameris M, et al. Lessons learnt from the first efficacy trial of a new infant tuberculosis vaccine since BCG. Tuberculosis (Edinb) 2013; 93: 143-149.

16. Fletcher HA, et al. T-cell activation is an immune correlate of risk in BCG vaccinated infants. Nature Communications 2016; 7: 11290.

17. Excler JL, Michael NL. Lessons from HIV-1 vaccine efficacy trials. Current Opinion HIV AIDS 2016; 11: 607-613.

18. Cohen J. Controversial HIV vaccine strategy gets a second chance. Science 2016; 354: 535.

19. Andrews JR, et al. Risk of progression to active tuberculosis following reinfection with Mycobacterium tuberculosis. Clinical Infectious Diseases 2012; 54: 784-791.

20. Kaufmann SH, Hussey G, Lambert PH. New vaccines for tuberculosis. Lancet 2010; 375: 2110-2119.

21. Barker LF, et al. Tuberculosis vaccine research: the impact of immunology. Current Opinion in Immunology 2009; 21: 331-338.

22. Abu-Raddad LJ, et al. Epidemiological benefits of more-effective tuberculosis vaccines, drugs, and diagnostics. Proceedings of the National Academy of Sciences of the United States of America 2009; 106: 13980-13985.

23. Knight GM, et al. Impact and cost-effectiveness of new tuberculosis vaccines in low- and middle-income countries. Proceedings of the National Academy of Sciences of the United States of America 2014; 111: 15520-15525.

24. Kaufmann SH, Evans TG, Hanekom WA. Tuberculosis vaccines: time for a global strategy. Science Translational Medicine 7: 276fs8.

25. Byrareddy SN, et al. Sustained virologic control in SIV+ macaques after antiretroviral and alpha4beta7 antibody therapy. Science 2016; 354: 197-202. 
26. Santangelo PJ, et al. Whole-body immunoPET reveals active SIV dynamics in viremic and antiretroviral therapy-treated macaques. Nature Methods 2015; 12: 427-432.

27. Coleman MT, et al. PET/CT imaging reveals a therapeutic response to oxazolidinones in macaques and humans with tuberculosis. Science Translational Medicine 2014; 6: $265 \mathrm{ra} 167$.

28. Lin PL, et al. PET CT identifies reactivation risk in cynomolgus macaques with latent $M$. tuberculosis. PLoS Pathogens 2016; 12: e1005739.

29. Daffe M, Etienne G. The capsule of Mycobacterium tuberculosis and its implications for pathogenicity. Tuberculosis and Lung Diseases 1999; 79: 153-169.

30. Prados-Rosales R, et al. The type of growth medium affects the presence of a mycobacterial capsule and is associated with differences in protective efficacy of BCG vaccination against Mycobacterium tuberculosis. Journal of Infectious Diseases 2016; 214: 426-437.

31. Lin PL, et al. The multistage vaccine $\mathrm{H} 56$ boosts the effects of BCG to protect cynomolgus macaques against active tuberculosis and reactivation of latent
Mycobacterium tuberculosis infection. Journal Clinical Investigation 2012; 122: 303-314.

32. Berry MP, et al. An interferon-inducible neutrophildriven blood transcriptional signature in human tuberculosis. Nature 2010; 466: 973-977.

33. Pulendran B. Learning immunology from the yellow fever vaccine: innate immunity to systems vaccinology. Nature Reviews Immunology 2009; 9: 741-747.

34. Ginsberg AM, et al. TB vaccines in clinical development. Tuberculosis (Edinburgh) 2016; 99(Suppl. 1): S16-S20.

35. Hawn TR, et al. Tuberculosis vaccines and prevention of infection. Microbiology Molecular Biology Reviews 2014; 78: 650-671.

36. Satti I, et al. Safety and immunogenicity of a candidate tuberculosis vaccine MVA85A delivered by aerosol in BCG-vaccinated healthy adults: a phase 1, doubleblind, randomised controlled trial. Lancet Infectious Diseases 2014; 14: 939-946.

37. Sheehan S, et al. A phase I, open-label trial, evaluating the safety and immunogenicity of candidate tuberculosis vaccines AERAS-402 and MVA85A, administered by prime-boost regime in BCG-vaccinated healthy adults. PLOS ONE 2015; 10: e0141687. 